\title{
Towards Evidence-Based Translational Research: The pros and cons of conducting systematic reviews of animal studies
}

Nijmegen, August 30, 2012

\author{
Judith van Luijk, Marlies Leenaars, Carlijn Hooijmans, Kim Wever, \\ Rob de Vries, and Merel Ritskes-Hoitinga
}

SYRCLE (SYstematic Review Centre for Laboratory animal Experimentation), Central Animal Laboratory, Radboud University Nijmegen Medical Centre (RUNMC), The Netherlands

Systematic reviewing (SR) is the methodological approach adopted by SYRCLE for the synthesis of evidence of animal studies. In addition to SRs of clinical trials, SRs of animal studies offer the important possibility of providing evidence on the potential translational value of animal models for humans. This mini symposium aimed at presenting the experiences of various researchers with performing systematic reviews of animal studies. The abstracts, presentations, photos, and videos of the mini symposium are available on the SYRCLE website: http://www. SYRCLE.nl. In this report we describe the main items presented during the mini symposium.

Several researchers shared their experiences with and results of performing SRs of animal studies. In addition, representatives of the Ministry of Health, Welfare and Sport and of ZonMW (The Netherlands Organisation for Health Research and Development) shed light on the concept and implementation of SRs of animal studies from a policy perspective. Moreover, methodology and implementation issues of performing SRs were part of the program as well.

The chair of the mini symposium was professor emeritus Bert van Zutphen (Department Animals in Science and Society, Division Animal Welfare and Laboratory Science, Utrecht University, The Netherlands).

Prof. Dr Melvin Samsom, Chair of the Board of the RUNMC opened the mini symposium and stressed the importance of SYRCLE in the chain of evidence-based healthcare by placing it in the context of the three main focus areas of the RUNMC: 1) high quality, 2) personalized medicine, and 3 ) efficiency and efficacy. He concluded his introduction by addressing the ethical aspects of animal use in biomedical research and the fundamental role evidence-based methodologies should play in this area.

Dr Henk Reinen (Ministry of Health, Welfare and Sport) gave a policy perspective on systematic reviews in research us- ing animals, in the context of "Better science with fewer animals." Starting from the motion in the Dutch Parliament, "SRs are standard in medical science and also should become the norm in science using animals," he discussed important topics that need attention in the short term. These included: raising support and awareness about SRs, change of culture, developing instruments, methodology, guidelines, and education.

Prof. Dr Maroeska Rovers (Department for Health Evidence, RUNMC) presented the parallels between SRs of clinical and of animal studies and the challenges that lie ahead for the latter category. She also mentioned what can be learned from the Cochrane Collaboration in this respect. Decades ago, human trials often were not randomized or blinded, as we still see today in publications of animal studies . The conduct of SRs helped make these shortcomings visible and thereby resulted in improved scientific practice. Rovers elaborated on the methodological challenges faced when performing SRs of animal studies. These include: the use of different animal models with varying comparability to the human condition, small groups with inadequate power, and limited attention to external validity (generalizability of the results). Items that deserve particular consideration are: comprehensive search strategies, analyses of individual animal data, real sample size calculations, demanding high quality standards in conducting and reporting of animal studies, multi-center animal studies, and establishing a register for animal studies in line with the clinical trial register.

MD Joris van Drongelen (Department of Obstetrics and Gynaecology, RUNMC) presented his findings and personal experiences when conducting the SR entitled "Effect of first pregnancy on vascular responses in mesenteric arteries". $\mathrm{He}$ illustrated the value of using SR for choosing an animal model, since it makes this process more evidence-based. In his SR, van Drongelen found that the Wistar and the Sprague Dawley rat demonstrate large vascular differences during pregnancy. This is 
valuable information when designing new animal experiments, as the animal model chosen will depend on the goal of the experiment. His advice and take home message was: "Systematic review of animal studies, just do it! There is no excuse."

Dr Carlijn Hooijmans (SYRCLE, RUNMC) has experience in conducting and supervising several SRs of animal studies at SYRCLE. In her presentation, she focused on the methodology of SRs of animal studies and explained the Whys and Hows. The items Hooijmans discussed included: 1) Searching systematically, 2) Critically appraising the included papers, and 3 ) data extraction and analysis (if possible, by a meta-analysis).

Dr Marlies Leenaars (SYRCLE, RUNMC) showed the activities of SYRCLE since 2008 aimed at facilitating and implementing SRs of animal studies. Leenaars focused on the following activities: raising (inter)national awareness, development of educational programs for animal researchers, $\mathrm{PhD}$, and master students, and the development of tools/guidelines to ease the process. Student evaluations demonstrate that education on SR of animal studies is highly valued.

Marije Sloff MSc (Department of Urology, RUNMC) just started her $\mathrm{PhD}$ project and shared her experiences with the SR she is currently conducting. Her SR is entitled: "What is the current evidence for the efficacy of tissue-engineered constructs for urinary diversion in animal models?" The three main reasons why she started her $\mathrm{PhD}$ with a SR are: 1) prevent unnecessary (animal) experimentation, 2) collect all relevant information about materials, methods, and models for this particular research, and 3) to generate a detailed overview of all relevant literature. Some difficulties she encountered were: 1) commercial side of her research (unpublished information), 2) negative results not published, 3) when to stop/continue developing the search strategy, and 4) the conduct of a SR can be a slow process. She concluded that the information she gained from the process and the product (a thorough literature overview) are worth the effort. Marije Sloff: "From the start of my PhD I have a good overview of available literature."

Linda Reus MSc (Department of Rehabilitation, RUNMC) studies the Prader Willy Syndrome (PWS). She started her SR to answer the following questions: 1) Can animal models provide more insight into neuromuscular functioning in PWS? 2) Is there a suitable animal model to study effects of training or medication on the neuromuscular system? She concluded that the SR has enabled her to make evidence-based decisions on which animal model is most suitable for her research.

Dr Kim Wever (Department of Surgery, RUNMC) studies the effects of ischemic preconditioning (IPC) in Ischemia Reperfusion Injury (IRI). She found that only one protocol for IPC is used in clinical trials, while many different protocols are being used in animal research. She started a SR to investigate what the optimal remote IPC protocol in humans would be, based on animal data. From the SR, she concluded that the current protocol used in the clinic probably can be improved. Overall, she concluded that SRs and meta-analysis of animal studies can: 1) contribute to improving clinical trial design, 2) contribute to improving animal study design, and 3) prevent unnecessary additional animal studies. Wever: "Translation of experimental data from animal studies into clinical practice is not straightforward. Systematic Reviews of animal studies can provide valuable clues to support this process."

Dr Erica van Oort (ZonMW; The Netherlands Organisation for Health Research and Development) presented the aims of the ZonMW program: More knowledge with fewer animals. She elaborated on Module Amendment 21 of this program, which involves: 1) support to publish negative results and stimulation of the use of the "Gold Standard Publication Checklist" , and 2) synthesis of evidence of animal experiments. ZonMW promotes synthesis of evidence by financially supporting education (national workshops) and consultation during the conduct of SRs by SYRCLE. Systematic review is the methodological approach SYRCLE has adopted for the synthesis of evidence.

Prof. Dr Bert van Zutphen concluded: "I learned a lot today, both on the advantages and disadvantages of SRs of animal studies. It took away some of my prejudices and convinced me that SRs do have an additional value to the current practice in animal research. Performing SRs of animal studies, however, should not be enforced but should become a natural routine."

\section{References}

Hooijmans, C., de Vries, R., Leenaars, M., et al. (2011). The Gold Standard Publication Checklist (GSPC) for improved design, reporting and scientific quality of animal studies GSPC versus ARRIVE guidelines. Lab Anim 45, 61.

Kilkenny, C., Parsons, N., Kadyszewski, E., et al. (2009). Survey of the quality of experimental design, statistical analysis and reporting of research using animals. PLoS One 4, e7824.

Pound, P. (2001). Scientific debate on animal model in research is needed. BMJ 323, 1252.

van Drongelen, J., Hooijmans, C. R., Lotgering, F. K., et al. (2012). Adaptive changes of mesenteric arteries in pregnancy: a meta-analysis. Am J Physiol Heart Circ Physiol 303, H639-657.

Wever, K. E., Menting, T. P., Rovers, M., et al. (2012). Ischemic preconditioning in the animal kidney, a systematic review and meta-analysis. PLoS One 7, e32296.

\section{Correspondence to}

Judith van Luijk

SYRCLE

Central Animal Laboratory

Radboud University Nijmegen Medical Centre (RUNMC)

The Netherlands

e-mail: SYRCLE@umcn.nl

http://www.SYRCLE.nl 